\title{
U(1) gauged Q-balls and their properties
}

\author{
Emin Nugaev ${ }^{1, \star}$, Alexander Panin ${ }^{1, \star \star}$, and Mikhail Smolyakov ${ }^{2,1, \star \star \star}$ \\ ${ }^{1}$ Institute for Nuclear Research of the Russian Academy of Sciences, \\ 60th October Anniversary prospect 7a, Moscow 117312, Russia \\ ${ }^{2}$ Skobeltsyn Institute of Nuclear Physics, Lomonosov Moscow State University, \\ Moscow 119991, Russia
}

\begin{abstract}
In the present manuscript, we briefly discuss the differences between ordinary Q-balls and U(1) gauged Q-balls. We focus on the differences in the existence of Q-ball solutions for appropriate values of the model parameters, as well as on the differences in classical stability of such Q-balls.
\end{abstract}

\section{Ordinary Q-balls}

Q-balls are localized solutions in the theory of a complex scalar field, described by the action

$$
S=\int d^{4} x\left(\partial^{\mu} \phi^{*} \partial_{\mu} \phi-V\left(\phi^{*} \phi\right)\right)
$$

such that

$$
\phi(t, \vec{x})=e^{i \omega t} f(r),\left.\quad f(r)\right|_{r \rightarrow \infty} \rightarrow 0,\left.\quad \partial_{r} f(r)\right|_{r=0}=0, \quad r=\sqrt{\vec{x}^{2}} .
$$

Without loss of generality one can take $f(r)$ to be real and such that $f(r)>0$. Initially, such solutions were proposed in [1] and became popular after the well-known paper [2].

The Q-ball charge is defined as

$$
Q=-i \int d^{3} x\left(\phi^{*} \dot{\phi}-\dot{\phi}^{*} \phi\right)=8 \pi \omega \int_{0}^{\infty} f^{2} r^{2} d r,
$$

whereas the Q-ball energy is defined as

$$
E=\int d^{3} x\left(\dot{\phi}^{*} \dot{\phi}+\partial_{i} \phi^{*} \partial_{i} \phi+V\left(\phi^{*} \phi\right)\right) .
$$

It is easy to show that the relation

$$
\frac{d E}{d Q}=\omega
$$

\footnotetext{
^e-mail: emin@ms2.inr.ac.ru

$\star \star$ e-mail: panin@ms2.inr.ac.ru

$\star \star \star$ e-mail: smolyakov@theory.sinp.msu.ru
} 
holds for Q-balls. Since there exists the symmetry

$$
\omega \rightarrow-\omega \quad \Rightarrow \quad Q \rightarrow-Q, \quad E \rightarrow E
$$

without loss of generality one can examine only the case $\omega \geq 0$.

As a simple example of the Q-ball, we can consider a model with the scalar field potential

$$
V\left(\phi^{*} \phi\right)=M^{2} \phi^{*} \phi \theta\left(1-\frac{\phi^{*} \phi}{v^{2}}\right)+M^{2} v^{2} \theta\left(\frac{\phi^{*} \phi}{v^{2}}-1\right)
$$

where $\theta$ is the Heaviside step function (see Figure 1). For Q-balls, this piecewise scalar field potential was introduced in a more general form in [1]. The corresponding solution for the scalar field takes the

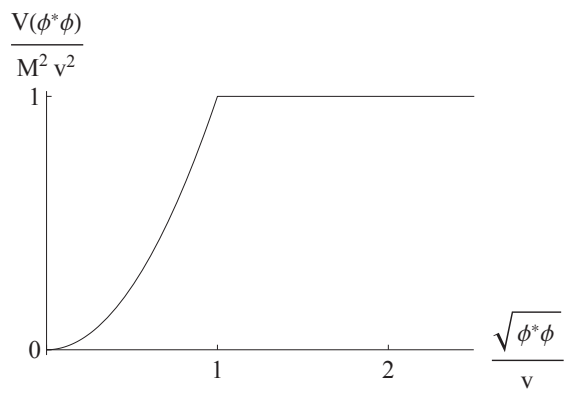

Figure 1. The scalar field potential (7).

form [1]

$$
\begin{array}{lll}
f=v R \frac{e^{-\sqrt{M^{2}-\omega^{2}}(r-R)}}{r}, & \text { for } \quad f<v \quad(r>R), \\
f=\frac{v R}{\sin (\omega R)} \frac{\sin (\omega r)}{r}, & \text { for } \quad f>v \quad(r<R) .
\end{array}
$$

with

$$
R=\frac{1}{\omega}\left(\arctan \left(-\frac{\omega}{\sqrt{M^{2}-\omega^{2}}}\right)+\pi\right), \quad 0<\omega<M .
$$

The solution for $\frac{\omega}{M}=0.8$ is presented in Figure 2. The $Q(\omega)$ and $E(Q)$ dependencies for these Q-balls are presented in Figure 3 (see [3] for a more detailed discussion of Q-balls in the model with the scalar field potential (7)).

\section{$2 \mathrm{U}(1)$ gauged Q-balls}

Now we turn to U(1) gauged Q-balls, which were initially proposed in [4], see also [5]. To describe such Q-balls, let us consider the action

$$
S=\int d^{4} x\left(\left(\partial^{\mu} \phi^{*}-i e A^{\mu} \phi^{*}\right)\left(\partial_{\mu} \phi+i e A_{\mu} \phi\right)-V\left(\phi^{*} \phi\right)-\frac{1}{4} F_{\mu \nu} F^{\mu \nu}\right) .
$$




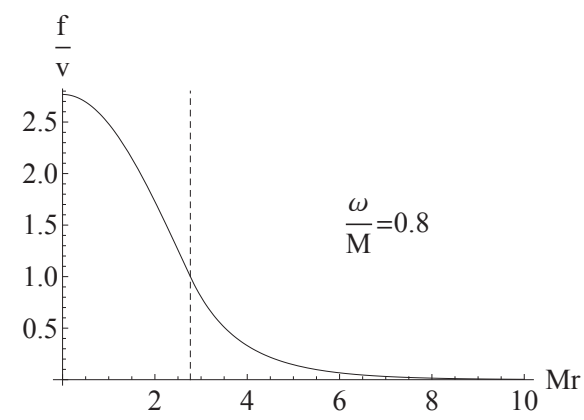

Figure 2. The scalar field profile for $\frac{\omega}{M}=0.8$. The vertical line indicates the point $r=R$ where $f(r)=v$.
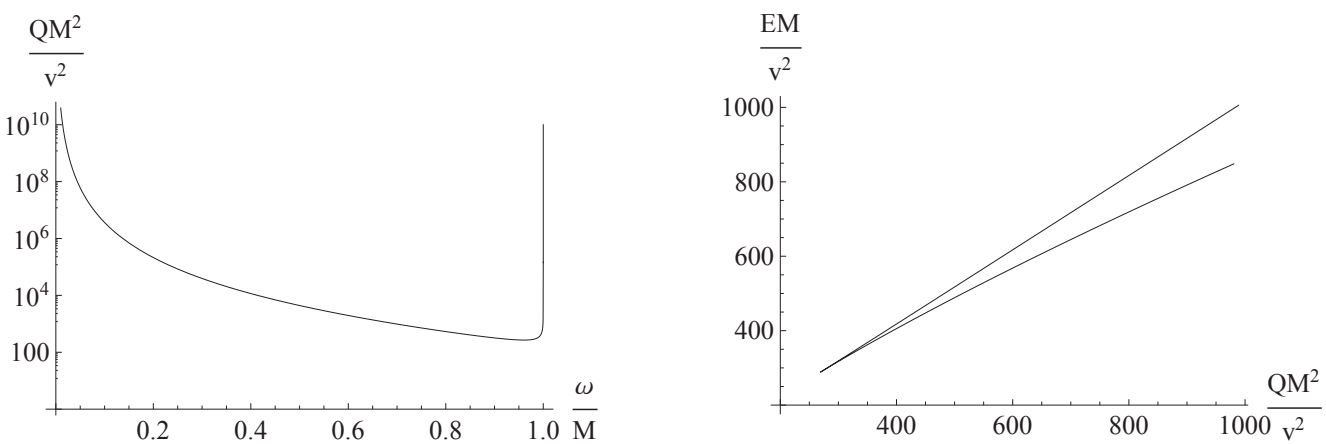

Figure 3. The $Q(\omega)$ and $E(Q)$ dependencies for the Q-ball defined by (8)-(10) (see [3] for details).

The U(1) gauged Q-ball is a localized solution to the equations of motion for the fields, following from action (11), of the form

$$
\begin{aligned}
\phi(t, \vec{x}) & =\mathrm{e}^{i \omega t} f(r), & \left.f(r)\right|_{r \rightarrow \infty} \rightarrow 0, & \left.\partial_{r} f(r)\right|_{r=0}=0, \\
A_{0}(t, \vec{x}) & =A_{0}(r), & \left.A_{0}(r)\right|_{r \rightarrow \infty} \rightarrow 0, & \left.\partial_{r} A_{0}(r)\right|_{r=0}=0, \\
A_{i}(t, \vec{x}) & \equiv 0 . & &
\end{aligned}
$$

Again, without loss of generality we take $f(r)>0$. The charge of U(1) gauged Q-ball is defined as

$$
Q=8 \pi \int\left(\omega+e A_{0}\right) f^{2} r^{2} d r
$$

whereas its energy is defined as

$$
E=4 \pi \int_{0}^{\infty}\left(\left(\omega+e A_{0}\right)^{2} f^{2}+\partial_{r} f \partial_{r} f+V(f)+\frac{1}{2} \partial_{r} A_{0} \partial_{r} A_{0}\right) r^{2} d r .
$$

It is possible to show [6] that the relation

$$
\frac{d E}{d Q}=\omega
$$


holds for U(1) gauged Q-balls too.

$\mathrm{U}(1)$ gauged Q-balls possess the following properties:

- $\omega \rightarrow-\omega \Rightarrow A_{0} \rightarrow-A_{0}$.

- The sign of $\omega+e A_{0}$ coincides with the sign of $\omega[5]$.

- If $A_{0} \equiv 0$, then $\omega=0$ [4].

Thus, there exists the symmetry

$$
\omega \rightarrow-\omega \Rightarrow Q \rightarrow-Q, \quad E \rightarrow E,
$$

and without loss of generality one can examine only the case $\omega \geq 0$.

After this brief introduction, we are ready to turn to examining the differences between ordinary Q-balls and U(1) gauged Q-balls.

\section{Behavior of Q-balls at $r \rightarrow \infty$}

Let us consider the scalar field potential $V\left(\phi^{*} \phi\right)$ such that

$$
V\left(\phi^{*} \phi\right) \rightarrow M^{2} \phi^{*} \phi \quad \text { for } \quad \phi^{*} \phi \rightarrow 0 .
$$

For $f(r) \rightarrow 0$, the equation of motion for ordinary Q-balls reduces to

$$
\left(\omega^{2}-M^{2}\right) f+\frac{1}{r} \frac{d^{2}}{d r^{2}}(r f) \approx 0,
$$

leading to $f(r) \sim \frac{e^{-\sqrt{M^{2}-\omega^{2} r}}}{r}$. It is clear that there are no Q-balls with $\omega=M$, because $Q \rightarrow \infty$ (as well as $E \rightarrow \infty)$ for $\omega \rightarrow M$.

In the case of $\mathrm{U}(1)$ gauged Q-balls the situation is completely different. Indeed, suppose that there exists a U(1) gauged Q-ball with the charge $Q$. Then, for $f(r) \rightarrow 0$ the equation of motion for the scalar field reduces to

$$
\left(\omega^{2}-M^{2}\right) f-\frac{2 \omega e^{2} Q}{4 \pi r} f+\frac{1}{r} \frac{d^{2}}{d r^{2}}(r f) \approx 0
$$

Now we have two cases:

- For $\omega<M$

$$
f(r) \sim \frac{\mathrm{e}^{-\sqrt{M^{2}-\omega^{2}} r}}{r^{1+\frac{\omega e^{2} Q}{4 \pi \sqrt{M^{2}-\omega^{2}}}}} \text { for } \quad r \rightarrow \infty .
$$

- For $\omega=M$

$$
f(r)=C \frac{K_{1}\left(\sqrt{\frac{2 M e^{2} Q}{\pi} r}\right)}{\sqrt{r}},
$$

where $C$ is a constant and $K_{1}(b, z)$ is the modified Bessel function of the second kind, leading to

$$
f(r) \sim \frac{\mathrm{e}^{-\sqrt{\frac{2 M e^{2} O}{\pi} r}}}{r^{\frac{3}{4}}} \text { for } r \rightarrow \infty .
$$

We see that in the gauged case there may exist a solution possessing the finite charge and energy even for $\omega=M$. This conclusion is supported by the results of numerical simulations (see [7] for details) for the scalar field potential (7) (Figures 4 and 5) and for the scalar field potential $V(f)=M^{2} f^{2}-\lambda f^{4}$ (Figures 6 and 7). 

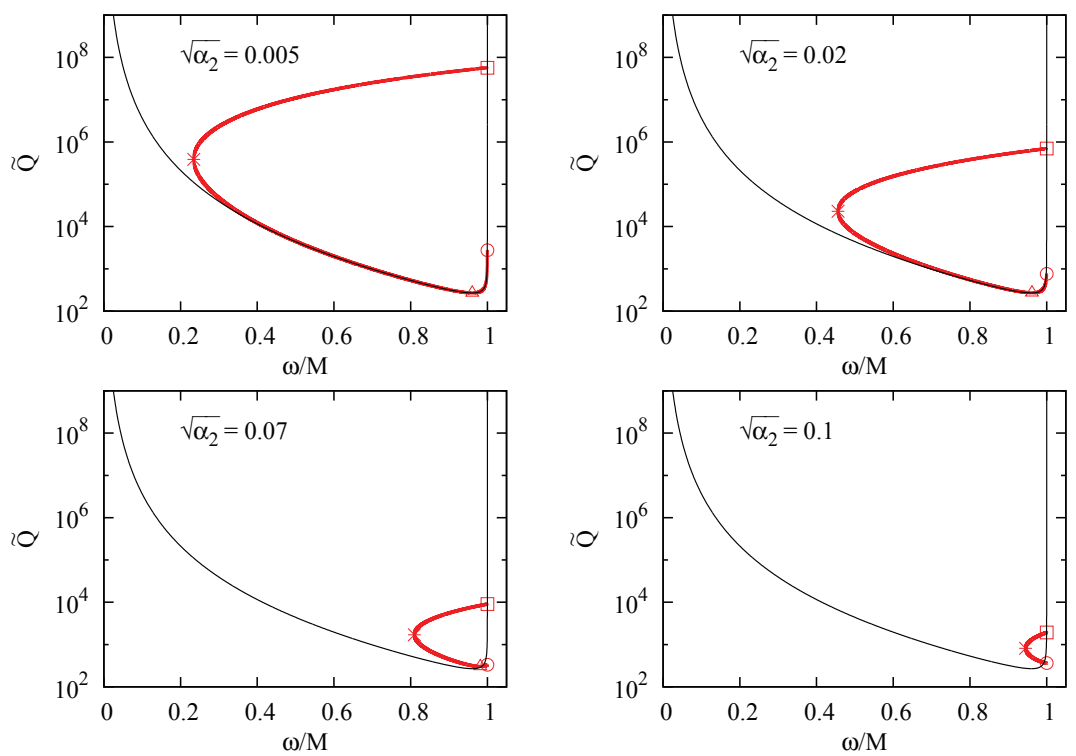

Figure 4. $\tilde{Q}=\frac{M^{2}}{v^{2}} Q$ for different values of the parameter $\alpha_{2}=\frac{e^{2} v^{2}}{M^{2}}$ (thick lines). The thin lines stand for the nongauged case. The figure is taken from [7].

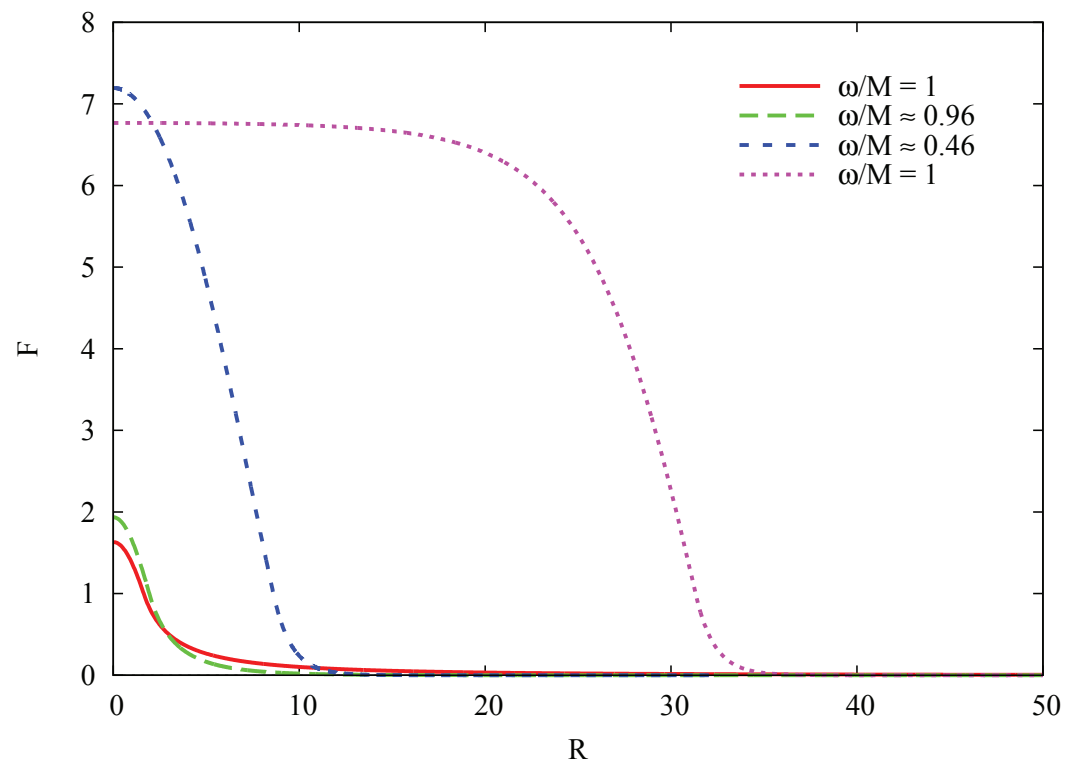

Figure 5. Profiles of the scalar field for different values of $\frac{\omega}{M}$. Here $\sqrt{\alpha_{2}}=0.02, R=M r, F(R)=\frac{1}{v} f(r)$. The figure is taken from [7]. 

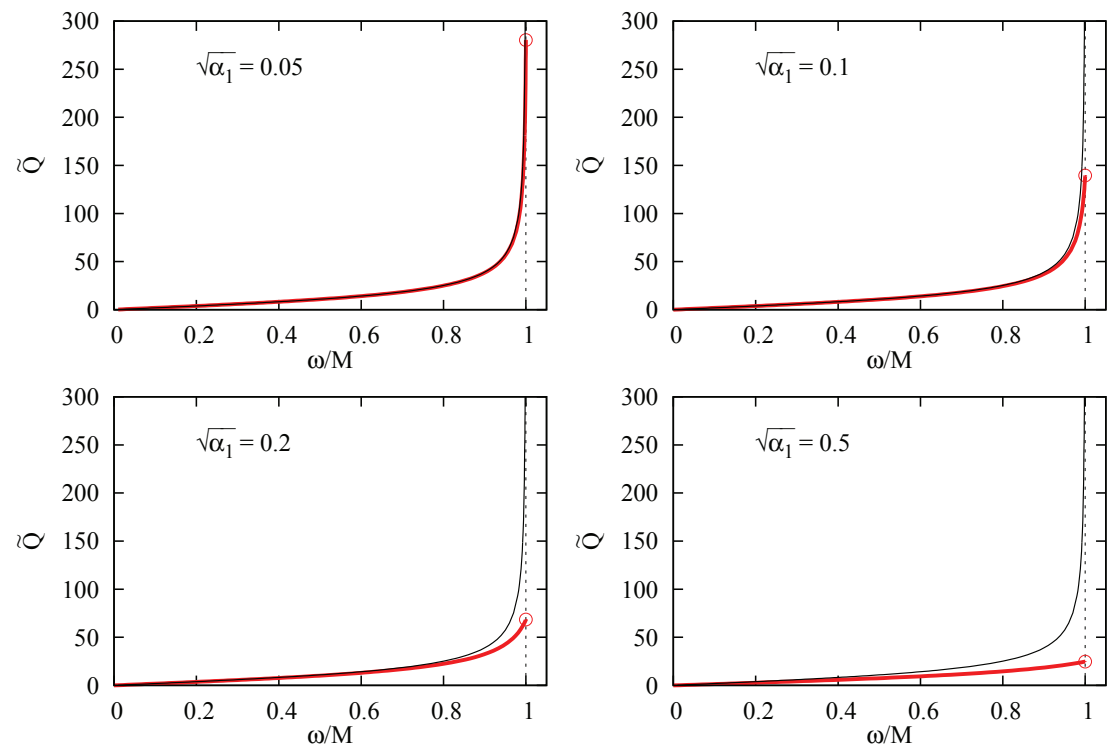

Figure 6. $\tilde{Q}=\lambda Q$ for different values of the parameter $\alpha_{1}=\frac{e^{2}}{\lambda}$ (thick lines). The thin lines stand for the nongauged case. The figure is taken from [7].

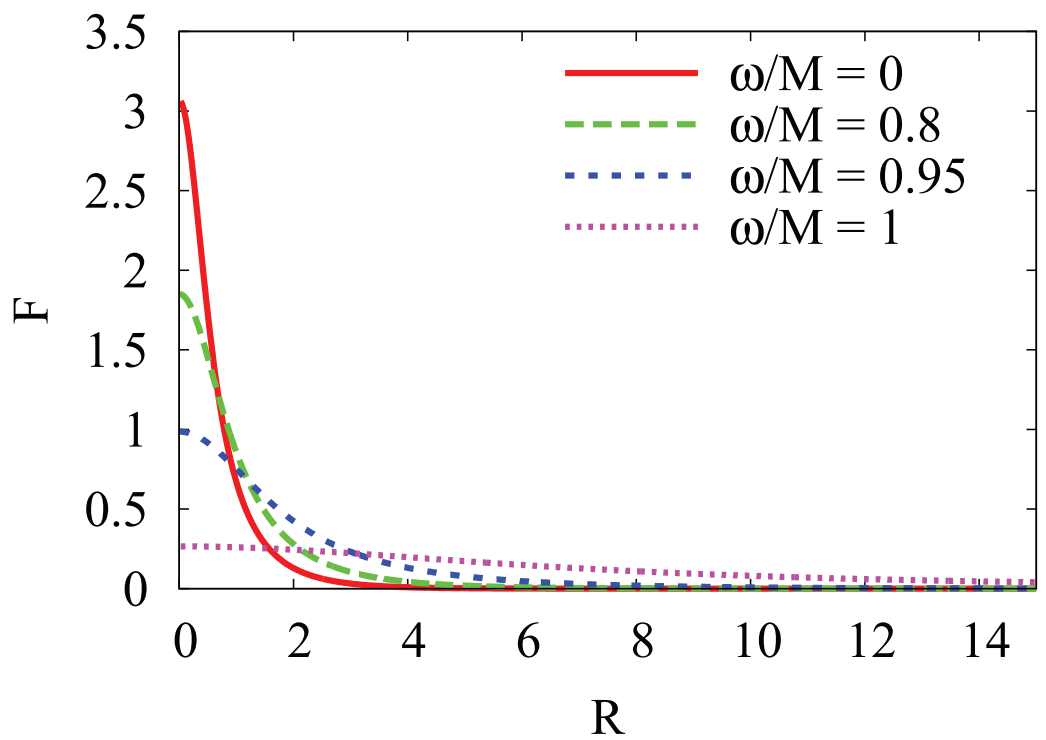

Figure 7. Profiles of the scalar field for different values of $\frac{\omega}{M}$. Here $\sqrt{\alpha_{1}}=0.05, R=M r, F(R)=\frac{\sqrt{\lambda}}{M} f(r)$. The figure is taken from [7]. 


\section{Classical stability of U(1) gauged Q-balls: theoretical considerations}

Now we turn to examining another difference between ordinary and U(1) gauged Q-balls - the classical stability, i.e., stability of the Q-ball solution with respect to small perturbations of the fields.

It is known that ordinary Q-balls are stable with respect to small perturbations if the following conditions hold:

1.

$$
\frac{d Q}{d \omega}<0
$$

2. The operator

$$
\hat{L}=-\Delta+\left.\frac{d V}{d\left(\phi^{*} \phi\right)}\right|_{\phi^{*} \phi=f^{2}(r)}+\left.2 \frac{d^{2} V}{d\left(\phi^{*} \phi\right)^{2}}\right|_{\phi^{*} \phi=f^{2}(r)} f^{2}(r)-\omega^{2},
$$

where $\Delta=\partial_{i} \partial_{i}$, has only one negative eigenvalue.

The proof of the classical stability criterion based on the use of the energy functional of the system can be found in [8], the proof based on the use of the linearized equations of motion along the lines of the Vakhitov-Kolokolov method proposed in [9] can be found in [10]. It should be noted that we are not aware of any explicit example of ordinary Q-ball, for which the operator $L$ has more than one negative eigenvalues and which is classically unstable for $\frac{d Q}{d \omega}<0$.

In order to examine the gauged case, we consider the perturbations above the U(1) gauged Q-ball solution in the form [10]

$$
\begin{aligned}
& \phi(t, \vec{x})=e^{i \omega t} f(r)+e^{i \omega t} e^{\gamma t}(u(\vec{x})+i v(\vec{x})), \\
& A_{0}(t, \vec{x})=A_{0}(r)+e^{\gamma t} a_{0}(\vec{x}), \\
& A_{i}(t, \vec{x})=e^{\gamma t} a_{i}(\vec{x}),
\end{aligned}
$$

where $\gamma$ is real, which passes through the linearized equations of motion. It is possible to show (see the detailed derivation in [10]) that U(1) gauged Q-balls are classically stable if the following conditions hold:

1.

$$
\frac{d Q}{d \omega}<0
$$

2. The corresponding operator $\hat{L}$ has only one negative eigenvalue.

Here the operator $\hat{L}$ takes the form

$$
\hat{L}=\left(\begin{array}{ccc}
-\Delta+W(r) & -2 e\left(\omega+e A_{0}\right) f & 0 \\
-2 e\left(\omega+e A_{0}\right) f & \frac{\Delta}{2}-e^{2} f^{2} & 0 \\
0 & 0 & \left(\frac{\Delta}{2}-e^{2} f^{2}-\frac{\gamma^{2}}{2}\right) I_{3 \times 3}
\end{array}\right),
$$

where $I_{3 \times 3}$ is the $3 \times 3$ unit matrix and

$$
W(r)=\left.\frac{d V}{d\left(\phi^{*} \phi\right)}\right|_{\phi^{*} \phi=f^{2}(r)}+\left.2 \frac{d^{2} V}{d\left(\phi^{*} \phi\right)^{2}}\right|_{\phi^{*} \phi=f^{2}(r)} f^{2}(r)-\left(\omega+e A_{0}\right)^{2} .
$$

One can see that there always exist negative eigenvalues of the operator $\hat{L}$, corresponding to the perturbations $a_{i}$ (because of the elements $\left.\left(\frac{\Delta}{2}-e^{2} f^{2}-\frac{\gamma^{2}}{2}\right) I_{3 \times 3}\right)$. Thus, the corresponding negative part 
of the spectrum is defined only by the spectrum of the operator $\left(\frac{\Delta}{2}-e^{2} f^{2}-\frac{\gamma^{2}}{2}\right)$. Moreover, due to the fact that $f(r)$ rapidly tends to zero at $r \rightarrow \infty$, one expects that the operator $\left(\frac{\Delta}{2}-e^{2} f^{2}-\frac{\gamma^{2}}{2}\right)$ has a negative continuous spectrum starting at $-\frac{\gamma^{2}}{2}$. Thus, fulfillment of the condition $\frac{d Q}{d \omega}<0$ does not provide any information about possible stability regions of the U(1) gauged Q-ball, at least within the framework of the generalized Vakhitov-Kolokolov approach used in [10]. At the moment it is not clear what is the origin of the problem described above. On the one hand, it is possible that the existence of the negative spectrum is just a technical artifact of the approach used in [10]. On the other hand, it is possible, due to the repulsive nature of the electromagnetic field, that nonspherically symmetric perturbations destroy any $U(1)$ gauged Q-ball. It is necessary to note that the question remains open as to whether it is possible to have only one negative eigenvalue of the operator $\hat{L}$ even in the case of spherically symmetric perturbations, in which the operator $\hat{L}$ takes a simpler form (see the detailed discussion in [10]).

\section{Classical stability of $U(1)$ gauged Q-balls: numerical simulations}

In order to examine the classical stability regions of $\mathrm{U}(1)$ gauged Q-balls, the numerical simulations in the spherically symmetric case were performed (see the details in [10]). Here we present the results for two different scalar field potentials. The first one is the potential (7). We see from Figure 8

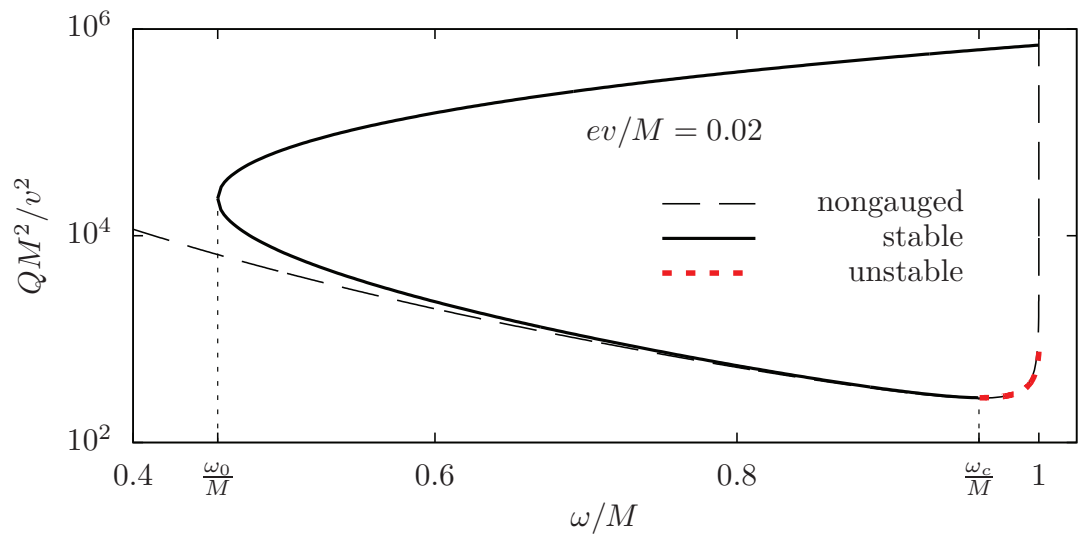

Figure 8. The stability and instability regions of Q-balls in the model with the scalar field potential (7). The longdashed line stands for the nongauged Q-balls. The solid line stands for the classically stable gauged Q-balls. The short-dashed line stands for the classically unstable gauged Q-balls. The figure is taken from [10].

that the inequality $\frac{d Q}{d \omega}>0$ holds for classically unstable solutions, which formally is in agreement with the classical stability criterion. Meanwhile, the inequality $\frac{d Q}{d \omega}>0$ also holds for gauged Qballs on the upper branch of the $Q(\omega)$ dependence, which appear to be stable at least with respect to spherically symmetric perturbations. Although formally this fact does not contradict the classical stability criterion (in the case of ordinary Q-balls the classical stability criterion does not guarantee that there exist unstable modes in the case $\frac{d Q}{d \omega}>0$, it just states that there are no classical instabilities if $\frac{d Q}{d \omega}<0$ ), this situation differs from the case of ordinary Q-balls, for which usually there exist spherically symmetric unstable modes for Q-balls with $\frac{d Q}{d \omega}>0$. 
The second scalar field potential has the form

$$
V\left(\phi^{*} \phi\right)=-\mu^{2} \phi^{*} \phi \ln \left(\beta^{2} \phi^{*} \phi\right) .
$$

In the nongauged case this scalar field potential was proposed in [11]. An interesting feature of this model is that the linearized equations of motion for perturbations can be solved exactly in the nongauged case [12]. The $\frac{d Q}{d \omega}<0$ stability criterion is valid for ordinary Q-balls in this model Q-balls with $\frac{d Q}{d \omega}<0$ are classically stable (and Q-balls with $\frac{d Q}{d \omega}>0$ are classically unstable).

The results of the numerical simulation in the gauged case can be found in Figure 9. One can easily
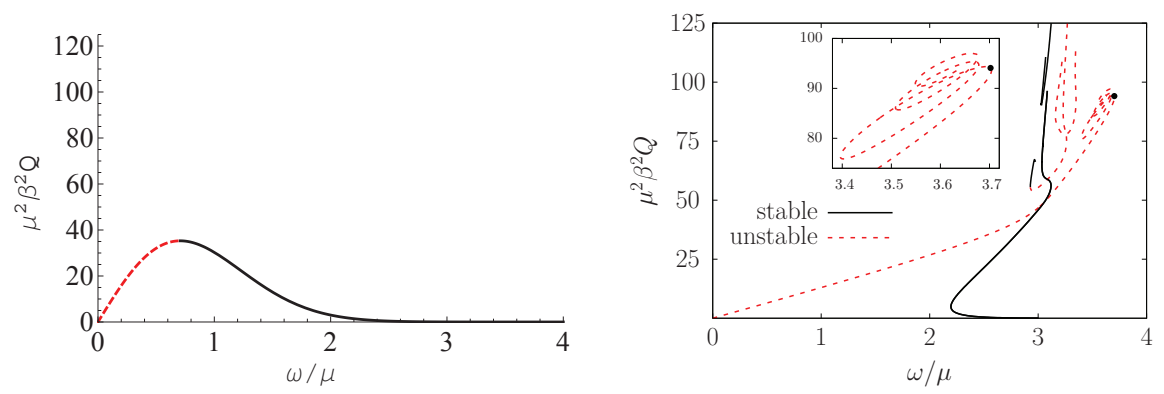

Figure 9. Ordinary (left plot) and $\mathrm{U}(1)$ gauged Q-balls for $e / \beta \mu=1.1$ (right plot) for the logarithmic scalar field potential. The solid lines stand for the classically stable Q-balls. The short-dashed lines stand for the classically unstable Q-balls. The right plot is taken from [10].

see from Figure 9 that there are classically unstable U(1) gauged Q-balls with $\frac{d Q}{d \omega}<0$ (for example, the Q-ball marked by the dot in Figure 9). This differs considerably from the case of ordinary Q-balls. An example of time evolution of the perturbed U(1) gauged Q-ball is shown in Figure 10, where the

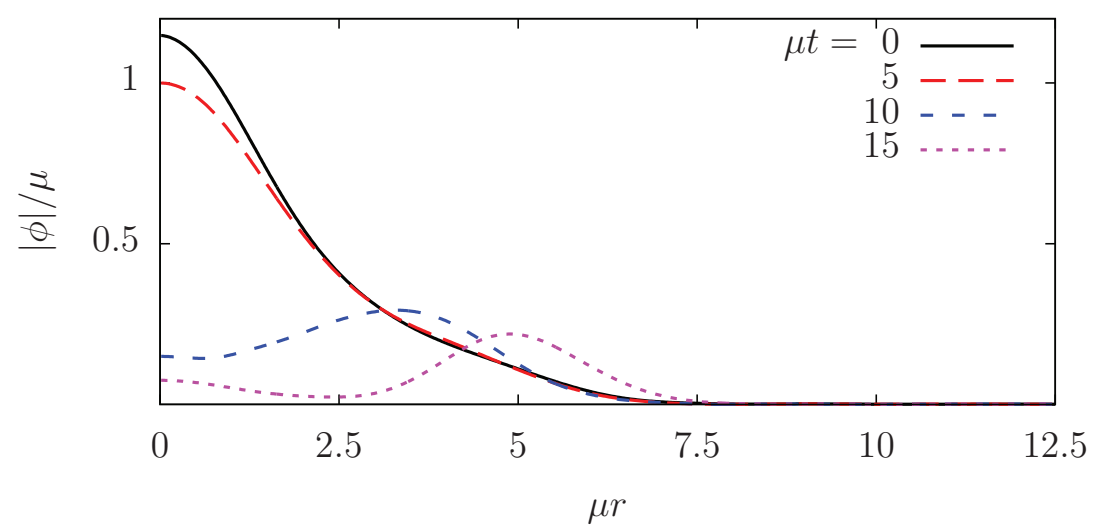

Figure 10. The scalar field profile of the classically unstable gauged Q-ball at different moments of time. The initial solution (at $\mu t=0$ ) is marked by the dot in the upper right corner of Figure 9. The figure is taken from [10].

scalar field profile is presented at different moments of time. 


\section{Conclusion}

In the present manuscript, we briefly discussed the differences between ordinary and U(1) gauged Q-balls. It is shown that $\mathrm{U}(1)$ gauged Q-balls may exist for the values of the parameters $(\omega=M)$, for which ordinary Q-balls do not exist. It is also shown that the classical stability criterion for ordinary Q-balls cannot be applied to U(1) gauged Q-balls. An explicit example of classically unstable U(1) gauged Q-ball with $\frac{d Q}{d \omega}<0$ is presented. These results demonstrate that addition of the gauge field changes drastically the properties of Q-balls.

The work was supported by the Grant 16-12-10494 of the Russian Science Foundation.

\section{References}

[1] G. Rosen, J. Math. Phys. 9, 996 (1968)

[2] S. R. Coleman, Nucl. Phys. B 262, 263 (1985) [Erratum-ibid. B 269, 744 (1986)]

[3] I. E. Gulamov, E. Y. Nugaev and M. N. Smolyakov, Phys. Rev. D 87, 085043 (2013)

[4] G. Rosen, J. Math. Phys. 9, 999 (1968)

[5] K.-M. Lee, J. A. Stein-Schabes, R. Watkins and L. M. Widrow, Phys. Rev. D 39, 1665 (1989)

[6] I. E. Gulamov, E. Y. Nugaev and M. N. Smolyakov, Phys. Rev. D 89, 085006 (2014)

[7] I. E. Gulamov, E. Y. Nugaev, A. G. Panin and M. N. Smolyakov, Phys. Rev. D 92, 045011 (2015)

[8] T. D. Lee and Y. Pang, Phys. Rept. 221, 251 (1992)

[9] N. G. Vakhitov and A. A. Kolokolov, Radiophys. Quantum Electron. 16, 783 (1973)

[10] A. G. Panin and M. N. Smolyakov, Phys. Rev. D 95, 065006 (2017)

[11] G. Rosen, Phys. Rev. 183, 1186 (1969)

[12] G.C. Marques and I.Ventura, Phys. Rev. D 14, 1056 (1976) 